\title{
EDITORIAL
}

\section{Polymer Journal for a forum on future polymer science}

\author{
Polymer Journal (2016) 48, 1-2; doi:10.1038/pj.2015.112
}

$\mathrm{I}$ wish you a Happy New Year. In 2016, we have started publishing volume 48. In the year of 2015, we published total 119 papers including 88 Original Articles, 2 Rapid Communications, 11 Notes, 14 Focus Reviews and 4 Invited Reviews. ${ }^{1}$ The access to the homepage of Polymer Journal (PJ; http://www.nature.com/pj/index.html) receives hundreds of thousand of accesses for every month. Focus Reviews that started in 2012 are written by many young talented polymer scientists. The focus review collection 2013-2015 collects a lot of attention. Please visit the homepage: http://www.nature.com/pj/focus/ focusreview2015.

Last year a new Associate Editor, Professor Takuma Yasuda, Kyushu University joined the editorial team. He is an expert of organic semiconductors and related functional polymer materials. Now we have six associate editors including Professor Wen-Chang Chen, National Taiwan University (functional polymers), Professor Kunio Kimura, Okayama University (polymer syntheses), Professor Shunsaku Kimura, Kyoto University (functional polymers), Professor Takeshi Serizawa, Tokyo Institute of Technology (bio-related polymers) and Professor Keiji Tanaka, Kyushu University (polymer physics and polymer interfaces; expertize in parenthes). This editorial team covers a wide range of fields that relates polymer science. We are trying to work hard for further development. We would like to have your suggestions for further improvement of the PJ.

We plan every year to organize one or two special issues. ${ }^{2-4}$ In the last year, the February issue was a special issue on fusion materials (Figure 1). ${ }^{2}$ The issue also received a large number of access and download. This special issue collects papers on materials produced by members of the MEXT (Ministry of Education, Culture, Sports, Science and Technology) project (https://www.fusion-materials.org/en/) entitled Fusion Materials: Creative Development of Materials and Exploration of Their Function through Molecular Control. This is the scheme of Grant-in-Aid for Scientific Research on Innovative Areas (2010-2014). You can also find a relevant special issue on self-assembled materials in no. $6,2012 .{ }^{3}$ In 2016, we are planning to publish a special issue on 'Polymer Surfaces, Interfaces and Thin films'; guest editors: Kookheon Char (Seoul National University), Wen-Chang Chen (National Taiwan University), Takeshi Serizawa (Tokyo Institute of Technology) and Keiji Tanaka (Kyushu University).

The impact factor, which is not the only measure for the quality of the journal, has increased from 1.554 in 2014 to 1.653 in 2015 (journal citation reports). In these 3 years, the most cited paper is the manuscript entitled design and synthesis of well-defined

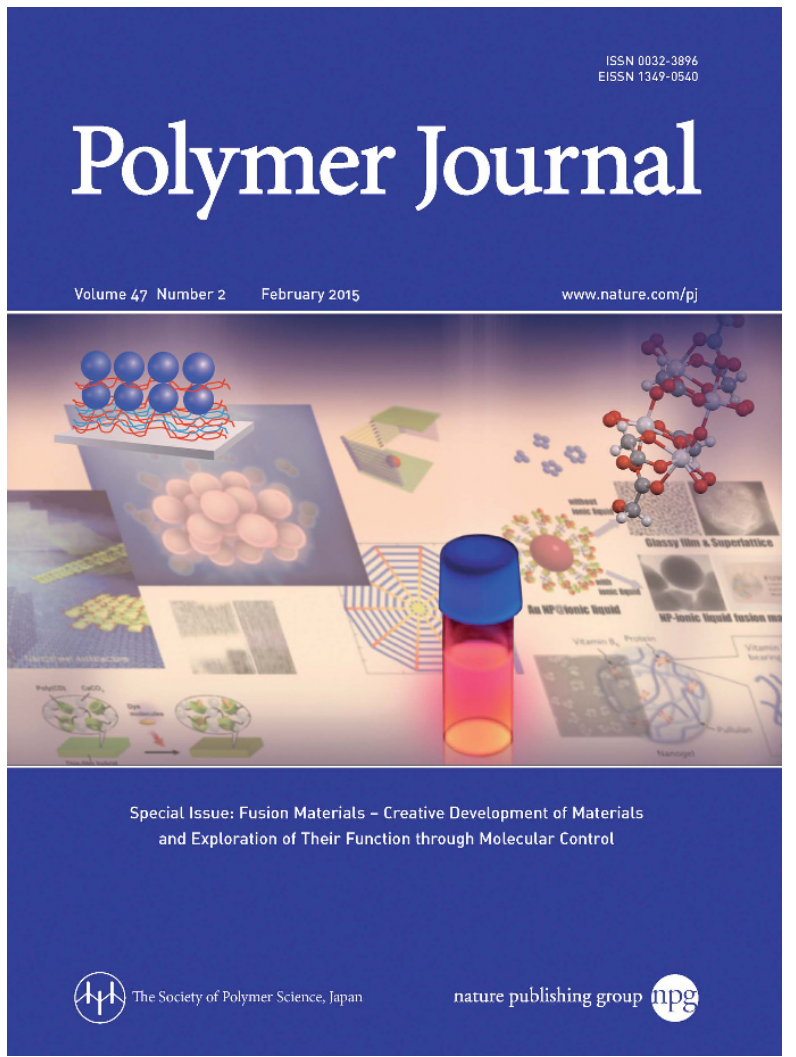

Figure 1 The cover of the February issue of 2015. The special issue of fusion materials.

glycopolymers for the control of biological functionalities by Yoshiko Miura, Kyushu University. ${ }^{5}$ I would like to thank these authors for submitting attractive papers. We will continue our efforts for making PJ more visible.

The PJ ZEON Award started since 2005 as the successor of the PJ Paper Award. ${ }^{6}$ (http://main.spsj.or.jp/c15/pjzaward/pjzjyuichiran-e. htm). The winners of the 2014 PJ ZEON Award have been announced by the Society of Polymer Science Japan (SPSJ) as follows: Tomoki Ogoshi (Kanazawa University, Japan), Xiaomei Shi (760th Research Institute China Shipbuilding Industry Corporation, China) and Syuhei Yamaguchi (Hitachi Zosen Corporation, Japan). 
Finally, I would also like to express my thanks to the readers, the referees, the Editorial and Advisory Board members, the Associate Editors, the Guest Editors and the staff of editorial and production offices of SPSJ and NPG for their present and future contributions to PJ. Allow me to take this opportunity to invite submission of your excellent papers for publication in $P J$, as we develop together new directions of the polymer sciences.
1 Kato, T. Polymer Journal for next generation of polymers and related materials. Polym. J. 47, 1 (2015).

2 Kato, T., Kikuchi, H. \& Imai, H. Special issue fusion materials: creative development of materials and exploration of their function through molecular control. Polym. J. 47, 77 (2015).

3 Kato, T., Aoshima, S. \& Kikuchi, H. Special issue: self-assembled materials. Polym. J. 44, 451 (2012).

4 Serizawa, T. Special issue: biorelated polymers and materials. Polym. J. 46, 435 (2014).

Takashi Kato 5 Miura, Y. Design and synthesis of well-defined glycopolymers for the control of biological functionalities. Polym. J. 44, 679-689 (2012).

Editor-in-Chief

Polymer Journal
6 Kato, T. PJ ZEON Award for outstanding papers in Polymer Journal 2014. Polym. J. 47, 413-414 (2015). 